EPJ manuscript No.

(will be inserted by the editor)

\title{
Jets in Nuclear Collisions: Status and Perspective *
}

\author{
Peter Jacobs \\ CERN and Lawrence Berkeley National Laboratory, e-mail: pmjacobs@lbl.gov \\ Received: date / Revised version: date
}

Abstract. I review the status and future directions of jet-related measurements in high energy nuclear collisions and their application as a probe of QCD matter.

PACS. $13.87 .-\mathrm{a}-25.75 . \mathrm{Nq}$

\section{Introduction}

Long before the startup of RHIC, jet physics was widely expected to play an important role in the study of QCD matter at collider energies. It is nevertheless surprising just how crucial a role that has turned out to be. Strong modification of jet fragmentation in high energy nuclear collisions is now well-established. The magnitude of the effects and the statistical abundance of jet-related ob' servables at RHIC have allowed characterization of this modification in significant detail, indicating that it arises mainly from interactions of high energy partons with the medium prior to hadronization. Perturbative QCD calculations incorporating partonic energy loss via mediuminduced gluon radiation ("jet quenching") are able to reproduce the broad features of the measurements, under the condition that the early hot phase of the collision fireball has gluon density 30-50 times that of cold nuclear matter. The jet quenching phenomena taken together with other measurements at RHIC, in particular evidence for early equilibration and near-ideal hydrodynamic flow, suggest that dense, locally equilibrated matter dominated by partonic degrees of freedom is created in high energy nuclear collisions [1,2, 3 .

These conclusions are at present somewhat qualitative, however. Important aspects of partonic energy loss theory are as yet untested. In particular, the induced radiation and its interaction with the medium have not been clearly identified, and the expected variation of energy loss with partonic species (gluon, light quark, heavy quark) is only beginning to be explored. Significant medium-induced effects in the "intermediate $p_{T}$ " regime at RHIC $\left(p_{T} \sim 2-5\right.$ $\mathrm{GeV} / \mathrm{c}$ ) have not yet been fully understood, though they appear to arise from the interaction between hard and soft processes and provide a new window into mechanisms that could drive the system to equilibrium.

This conference is therefore timely. The qualitative discovery period for jet physics in nuclear collisions at RHIC

\footnotetext{
* Summary talk on jet physics, Hard Probes 2004, Ericeira, Portugal, Nov. 4-10 2004
}

is perhaps winding down and the most significant progress may now come from quantitative comparison of measurement and theory. I will summarize some of the main experimental results to date, highlighting recent developments presented at this conference, and briefly look ahead to the RHIC II and LHC eras. The field of partonic interactions in matter is large and growing and there are important areas I will not cover, for instance forward physics which is discussed in 5 .

\section{Hadron Suppression and Partonic Energy Loss}

The inclusive $p_{T}$ spectrum in a high energy hadronic collision is dominated by hadrons carrying a large fraction of the energy of their parent partons $(\langle z\rangle \sim 0.7$ for the inclusive hadron spectrum compared to $\langle z\rangle \sim 0.3$ for the highest energy hadron in a light quark jet, where $z=$ $\left.p_{\text {hadron }} / E_{\text {jet }}\right)$. Modification of the partonic spectrum due to energy loss in matter is therefore reflected directly in the suppression of inclusive hadron yields at high $p_{T}$. Strong medium-induced suppression (factor $\sim 5$ ) has been measured in nuclear collisions at RHIC, providing the primary evidence for partonic energy loss [1,2,3,4]. In contrast, prompt photon production has been observed not to be suppressed in nuclear collisions [6], consistent with the interpretation of high $p_{T}$ hadron suppression resulting from the interaction of colored partons in the medium.

Figure 1 shows recent measurements of hadron suppression in nuclear collisions from PHENIX [7]. $R_{A A}\left(p_{T}\right)$ is the ratio of the inclusive yield in nuclear collisions to the yield in $\mathrm{p}+\mathrm{p}$ collisions, scaled by the number of binary collisons $\left\langle N_{b i n}\right\rangle$ to remove uninteresting geometric effects. $R_{A A}\left(p_{T}\right)=1$ correpsonds to no nuclear modification. The well-known factor $\sim 5$ suppression of $\pi^{0}$ now extends to $p_{T}=14 \mathrm{GeV} / \mathrm{c}$, showing no significant $p_{T}$-depdendence. New at this conference is $R_{A A}\left(p_{T}\right)$ for $\eta$ mesons, whose suppression is in quantitative agreement with that for $\pi^{0}$. The $\eta / \pi^{0}$ yield ratio is independent of collision system 


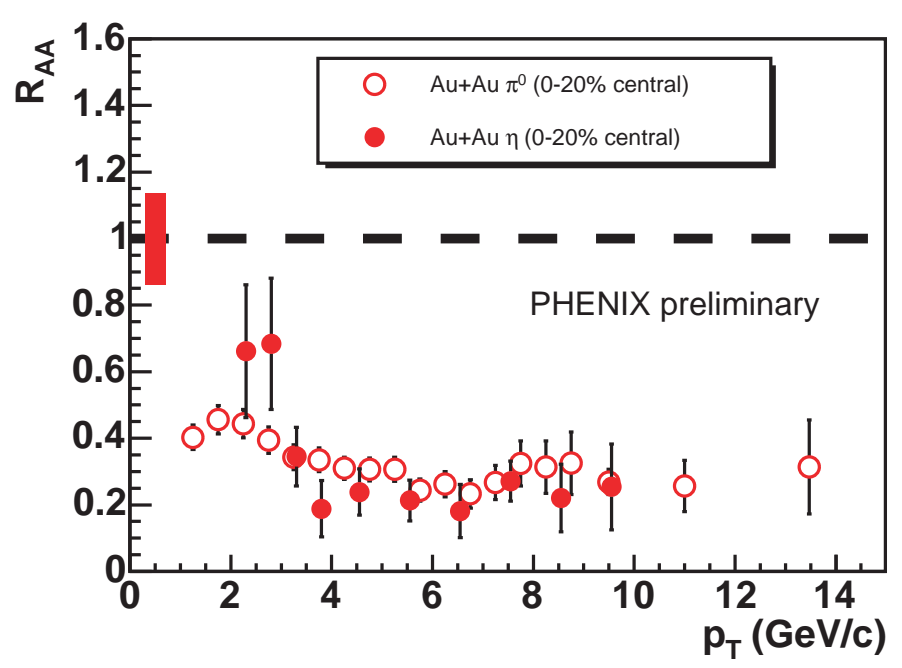

Fig. 1. Binary collision-scaled ratio $R_{A A}\left(p_{T}\right)$ of $\pi^{0}$ and $\eta$ inclusive yields, for central $\mathrm{Au}+\mathrm{Au}$ relative to $p+p$ collisions 7.

[7, consistent with hadron production being dominated by jet fragmentation in vacuum even for central $\mathrm{Au}+\mathrm{Au}$ collisions. Figure 2 left panel, shows the equally wellknown $R_{A A}\left(p_{T}\right)$ for charged hadrons in central $\mathrm{Au}+\mathrm{Au}$ collisions, whose suppression for $p_{T}>5 \mathrm{GeV} / \mathrm{c}$ is in quantitative agreement with that for $\pi^{0}$. The growth toward lower $p_{T}$ for charged hadron $R_{A A}\left(p_{T}\right)$ arises from different suppression for protons and pions in this region; more on this point below.

The inclusive $\pi^{0}$ spectrum in $200 \mathrm{GeV} \mathrm{p}+\mathrm{p}$ collisions is well represented by NLO pQCD calculations incorporating suitable fragmentation functions [8], meaning that the reference spectrum for the inclusive suppression measurements is well understood. Medium-induced radiative energy loss has been incorporated into factorized pQCD calculations in two approximations, multiple soft interactions (BDMPS) and few hard scatterings (GLV opacity expansion), and the modification of the fragmentation function measured in DIS scattering off nuclei has been related to its modification in heavy ion collisions via a twist expansion (Wang and Wang) (for recent reviews see 9,10,11]). Within the BDMPS framework the medium is characterized by a transport coefficient $\hat{q}=\left\langle q_{T}^{2}\right\rangle / \lambda$, where $\left\langle q_{T}^{2}\right\rangle$ is the mean squared transverse momentum generated by interactions in the medium over mean free path $\lambda$. $\hat{q}$ is related to the energy density via $\hat{q}=c \epsilon^{3 / 4}$, where $c \sim 2$ for an ideal pion gas and weakly interacting quark-gluon plasma [12] but takes values $c \sim 10-20$ from estimates based on hydrodynamics 13 . In the high energy limit for a static medium of spatial dimension $L$ the average radiative energy loss is $\Delta E \sim \alpha_{s} \hat{q} L^{2} / 2$ [12, though this expression is only qualitative since finite kinematic limits and dynamic expansion effects generate significant modifications to the radiated spectrum and reduce the $L$ dependence to linear. However, the induced radiation spectrum for a dynamically expanding medium is the same as that for a static medium having $\hat{q}$ equal to the time-averaged $\hat{q}$ for the dynamic case 14 .
With suitable choice of model parameters, the multiple soft scattering and opacity expansion approximations generate similar effects for energy loss and jet broadening 14, and calculations applying either approach are successful in describing the inclusive hadron suppression and its systematic dependence on centrality and collision energy [15 16]. Figure 2 right panel, shows $R_{A A}\left(p_{T}\right)$ calculated in the BDMPS framework compared to the data in the left panel 13 . For $p_{T}>\sim 5 \mathrm{GeV} / \mathrm{c}$ the level of suppression is described equally well by all choices $\hat{q}>5$ $\mathrm{GeV}^{2} / \mathrm{fm}$, due to the complete dissipation of the energy of hard-scattered partons in the core of the fireball for large $\hat{q}$ [13. In this and similar calculations the observed hadron population at high $p_{T}$ is biased towards fragments of partons scattered near the surface of the fireball and directed outward, thereby suffering relatively little energy loss (see also [17,18,19]).

Inclusive hadron production is therefore subject not only to fragmentation bias (prefering quark to gluon jets) but also to geometric bias towards peripheral production and small energy loss. Its information content is consequently limited: for a core that is sufficiently opaque, measurement of the leading hadron suppression has little sensitivity to just how opaque it is [13] and study of partonic interactions with the medium must rely on additional measurements, in particular correlations.

Figures 1 and 2 show that $R_{A A}\left(p_{T}\right)$ at high $p_{T}$ is largely independent if $p_{T}$. While several pQCD-based calculations incorporating radiative energy loss are able to reproduce the $p_{T}$-independence of the suppression, they ascribe it variously [21] to the interplay between quenching, shadowing, and Cronin enhancement 22, absorption of thermal gluons at moderate $p_{T}$ 23, the dominance of geometry due to strong absorption [19.13, and the interplay between energy loss and steepening of the partonic spectrum due to phase space limitations [13. It is important to disentangle these mechanisms, for instance to isolate the effects of stimulated emission and absorption which can drive parton thermalization 21]. Variation of $\sqrt{s_{\mathrm{NN}}}$ provides a potentially signficiant test of the models: lowering $\sqrt{s_{\mathrm{NN}}}$ steepens the underlying partonic spectrum which is expected to generate stronger Cronin enhancement and stronger hadron suppression at fixed energy loss, while the initial gluon density of the medium may be lower, resulting in reduced energy loss.

In 2004 RHIC carried out a two week run of $\mathrm{Au}+\mathrm{Au}$ at $\sqrt{s_{\mathrm{NN}}}=62.4 \mathrm{GeV}$, chosen to match the top ISR energy where extensive $\mathrm{p}+\mathrm{p}$ reference data are available. It turns out, unfortunately, that disagreement among the ISR datasets limits the systematic uncertainty of the $\mathrm{p}+\mathrm{p}$ reference to about $30 \%$ [24,25]. Nevertheless, strong suppression of $\pi^{0}$ and charged hadron inclusive yields is observed in $62 \mathrm{GeV} \mathrm{Au}+\mathrm{Au}$ central collisions for $p_{T}>\sim 5$ $\mathrm{GeV} / \mathrm{c}$, beyond the baryon enhancement region [26 25]. In particular, the binary scaled central to peripheral ratio $R_{C P}\left(p_{T}\right)$, which does not rely on the ISR reference, exhibits the same magnitude of suppression for 62 and $200 \mathrm{GeV} \mathrm{Au}+\mathrm{Au}$ collisions 25]. The measured high $p_{T}$ suppression at $62 \mathrm{GeV}$ broadly agrees with theoretical 

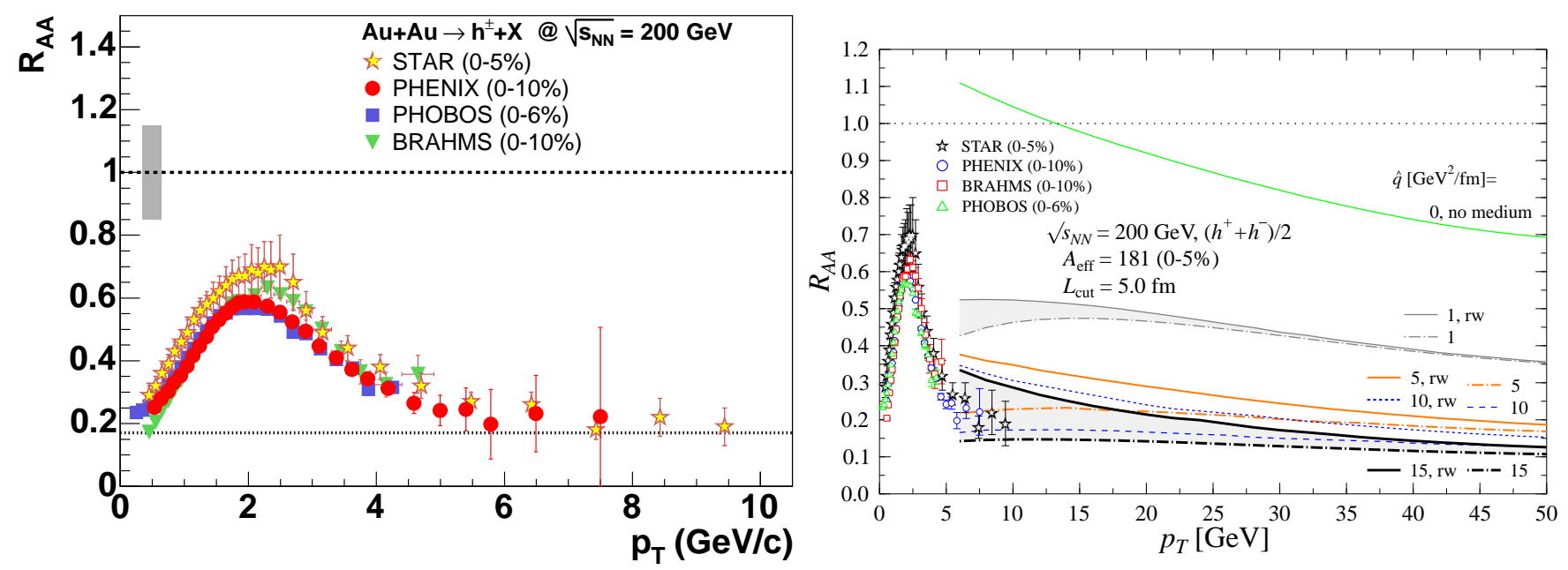

Fig. 2. Left: $R_{A A}\left(p_{T}\right)$ in central Au+Au collisions for charged hadrons [20]. Right: the same data compared to a BDMPS-based calculation with varying transport coefficient $\hat{q}[13$. The pair of curves for each $\hat{q}$ indicates the theoretical uncertainty due to finite energy corrections ("rw" refers to reweighting the gluon emission probability distribution so that the total radiated energy does not exceed the energy of the parent parton).

predictions 19,24,27,28,29, though the limited statistical reach of the current $62 \mathrm{GeV}$ dataset does not support more quantitative conclusions. The RHIC program will return to $62 \mathrm{GeV} \mathrm{Au}+\mathrm{Au}$ for a longer run and additionally will acquire its own high statistics $\mathrm{p}+\mathrm{p}$ reference data at $62 \mathrm{GeV}$.

For SPS fixed target data at yet lower $\sqrt{s_{\mathrm{NN}}} \sim 17 \mathrm{GeV}$, reconsideration of the available $\mathrm{p}+\mathrm{p}$ reference data brings $\pi^{0}$ suppression into agreement with partonic energy loss calculations incorporating Bjorken model estimates of the energy density [24, though the statistics in the hard scattering region are quite limited. Overall, precision study of the $\sqrt{s_{\mathrm{NN}}}$ dependence of inclusive hadron suppression remains open.

\section{Jet-like Correlations}

Hard partonic scattering generates pairs of recoiling jets which are to first order back-to-back in azimuth. While full jet reconstruction is challenging in high energy nuclear collisions due to the large combinatorial background, additional insight into partonic energy loss can be gained by studying jet-like correlations of high $p_{T}$ pairs ("dihadrons"). Figure 3 left panel, shows the dihadron distribution in relative azimuthal angle $(\Delta \phi)$, with trigger $p_{T}{ }^{\text {trig }}>4 \mathrm{GeV} / \mathrm{c}$ and associated hadron $2<p_{T}<p_{T}{ }^{\text {trig }}$ [30]. Dihadrons at small relative angle $\Delta \phi \sim 0$ are drawn from the same jet cone. The geometric bias discussed above should also affect the dihadron trigger, and the approximate similarity of the $\Delta \phi \sim 0$ correlation seen for $\mathrm{p}+\mathrm{p}$, $\mathrm{d}+\mathrm{Au}$ and central $\mathrm{Au}+\mathrm{Au}$ collisions is consistent with negligible partonic energy loss and fragmentation in vacuum in the latter case.

Back-to-back dihadrons $(\Delta \phi \sim \pi)$ are drawn from the mutually recoiling jet pair. The presence of back-to-back correlations in $\mathrm{p}+\mathrm{p}$ and $\mathrm{d}+\mathrm{Au}$ but their absence in central $\mathrm{Au}+\mathrm{Au}$ collisions suggests strong suppression of the leading fragments of the recoiling jet, consistent with significant medium-induced partonic energy loss. The geometric bias of the trigger also plays an important role in this case: since the trigger is biased towards short path length in the medium, the recoil is biased towards large path length through the core of the fireball, thereby enhancing the energy loss effects.

The observed suppression of back-to-back dihadrons at high $p_{T}$ is sometimes mis-stated as the suppression of back-to-back jets. The recoil jet has not been suppressed, rather its fragmentation has apparently been softened. Its energy and momentum should be conserved and should reappear at lower $p_{T}$. This has been demonstrated qualitatively in 31, where the soft recoiling hadrons in central collisions are seen to be distributed somewhat more broadly in azimuth and softened in $p_{T}$ relative to similar distributions in $\mathrm{p}+\mathrm{p}$, with the azimuthal distribution consistent with no dynamical correlations beyond simple momentum conservation 32. There has recently been discussion of additional structure in the recoil azimuthal distribution at low $p_{T}$ which may be generated, for instance, by sonic shock waves resulting from deposition of the recoiling jet energy in an ideal, low-viscosity fluid 33. Such measurements require careful subtraction of background, however, and the significance of the features seen in the data is under discussion.

Figure 3 right panel, shows a similar high $p_{T}$ dihadron analysis for non-central $\mathrm{Au}+\mathrm{Au}$ collisions, with the trigger now constrained to lie within restricted azimuthal intervals centered on the reaction plane orientation or orthogonal to it 34. The small angle $\Delta \phi \sim 0$ correlation is seen to be independent of the orientation of the trigger while the recoil $\Delta \phi \sim \pi$ correlation is sensitive to it, with stronger suppression out-of-plane. Again taking into account the 

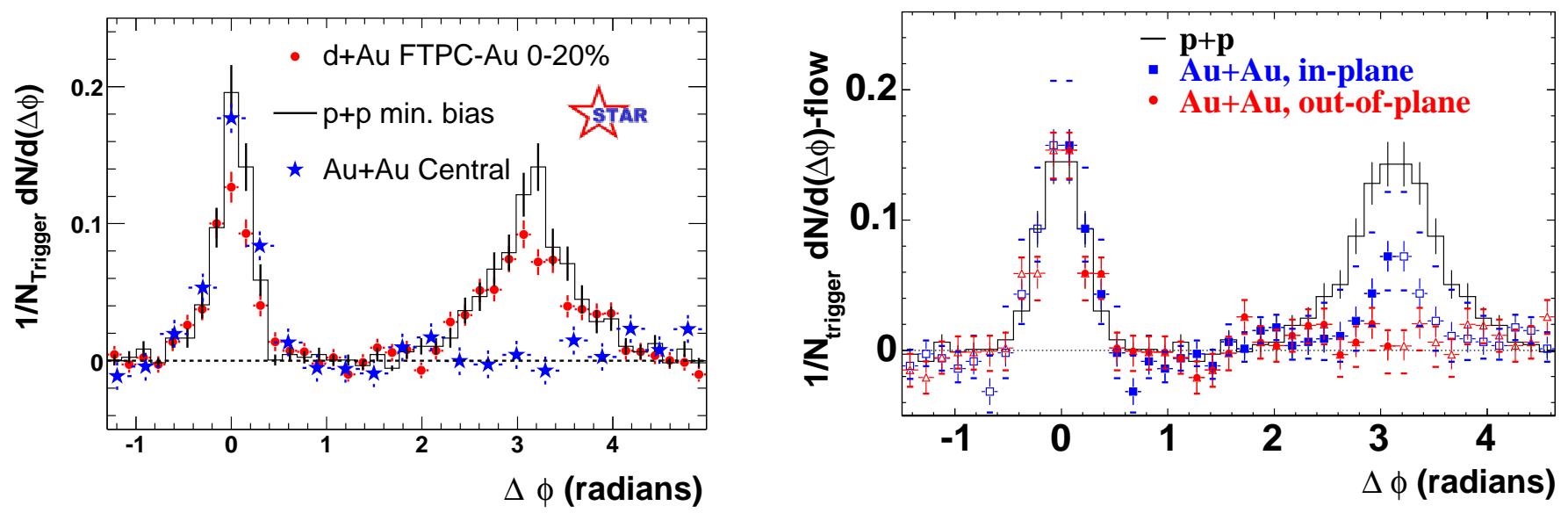

Fig. 3. Relative azimuthal angle distribution for high $p_{T}$ dihadrons at $\sqrt{s}=200 \mathrm{GeV}$. Left: $\mathrm{p}+\mathrm{p}, \mathrm{d}+\mathrm{Au}$ and central $\mathrm{Au}+\mathrm{Au}$ collisions 30. Right: trigger particle in or out of reaction plane in non-central Au+Au collisions 34.

geometric trigger bias, an out-of-plane trigger corresponds to larger in-medium path length for the recoil than an inplane trigger. This is the clearest indication to date of the in-medium path length dependence of hadron suppression. Though the systematic uncertainties in the figure are large and strongly (anti-)correlated between the trigger selections, this is nevertheless a promising observable for the high luminosity RHIC Run 4 analysis, where the study of higher $p_{T}$ correlations with correspondingly smaller background corrections may enable a detailed measurement of the path length dependence of the suppression.

One of the striking observations at RHIC is the large medium-induced enhancement of the baryon/meson inclusive yield ratio in the intermediate $p_{T}$ region $\sim 2<p_{T}<5$ $\mathrm{GeV} / \mathrm{c}$ 35.36, which reverts at higher $p_{T}$ to the ratio observed for jet fragmentation in vacuum. Stated differently, mesons at intermediate $p_{T}$ exhibit strong suppression while baryons are much less suppressed, scaling roughly as the number of binary collisions. Measurements of several hadron species indicate that this is a meson/baryon distinction, not dependent upon particle mass 25/37. Additionally, elliptic flow $v_{2}$ (the azimuthal modulation of the inclusive yield relative to the reaction plane) is seen to be large at intermediate $p_{T}$ in non-central collisions, apparently exceeding the initial spatial anisotropy 34, with $v_{2}$ for baryons larger than that for mesons 36. The large $v_{2}$ values cannot be fully accounted for by so-called "nonflow" contributions (e.g. intra-jet correlations at high $p_{T}$ ) 34. It is difficult to reconcile these observations with a scenario in which hadron production results dominantly from jet fragmentation and its modification due to partonic energy loss: stronger suppression of inclusive meson than baryon distributions would correspond to larger energy loss in medium and therefore larger azimuthal modulation $v_{2}$ for mesons, in contrast to the measurements.

An alternative view of hadron production at intermediate $p_{T}$ emerges from the observation that $v_{2}$ for various meson and baryon species approximately follows a common distribution when rescaled as $v_{2} / n$ vs $p_{T} / n$ 36. 25], where $n=3$ for baryons and $n=2$ for mesons.
Such scaling is expected from models in which hadronization occurs via coalescence or recombination of constituent quarks 38,39,40,41. The models consider recombination of dressed quarks from two origins: a collectively flowing thermal medium generating an exponential $p_{T}$ distribution, and fragmentation of hard scattered partons generating a power law $p_{T}$ distribution. Because baryons carry the momentum of three recombining quarks compared to two for mesons, generically in these models the thermal component is dominant to higher hadronic $p_{T}$ for baryons than mesons. The enhancement at intermediate $p_{T}$ of the baryon/meson inclusive yield ratio, the larger baryon $v_{2}$, and the constituent quark number scaling of $v_{2}$ emerge naturally from the recombination approach.

Hadron production via quark coalescence from a thermalized medium will generate different correlation structure than production via parton fragmentation, and dihadron correlation measurements therefore provide additional tests of the recombination picture. The left panels of Fig. [- 1 show measurements by PHENIX of the correlated yield measured at small angular separation ("near side") or back-to-back ("away side") relative to triggers identified as pion or proton 42. Significant correlated yield is seen for both trigger classes, in particular on the near side where the correlation is stronger for $\mathrm{Au}+\mathrm{Au}$ collisions than for the $\mathrm{d}+\mathrm{Au}$ and $\mathrm{p}+\mathrm{p}$ reference systems. The dashed line shows results of a calculation in which thermal recombination is responsible for all excess baryons, inadequate to account for the observed correlation strength.

The right panels in Fig. [4 show similar measurements from STAR for identified $\Lambda$ and $K_{s}^{0}$ triggers in the same kinematic window 43 44, confirming the PHENIX observation of similar associated yield for meson and baryon triggers. (The numerical differences in correlation strength between the measurements may be attributable to their different $\eta$ acceptances.) The trigger $p_{T}$ interval in these measurements corresponds to intermediate $p_{T}$, where there is a marked difference in the suppression of meson and baryon inclusive yields in more central collisions. The nearside associated yield is therefore largely independent of 

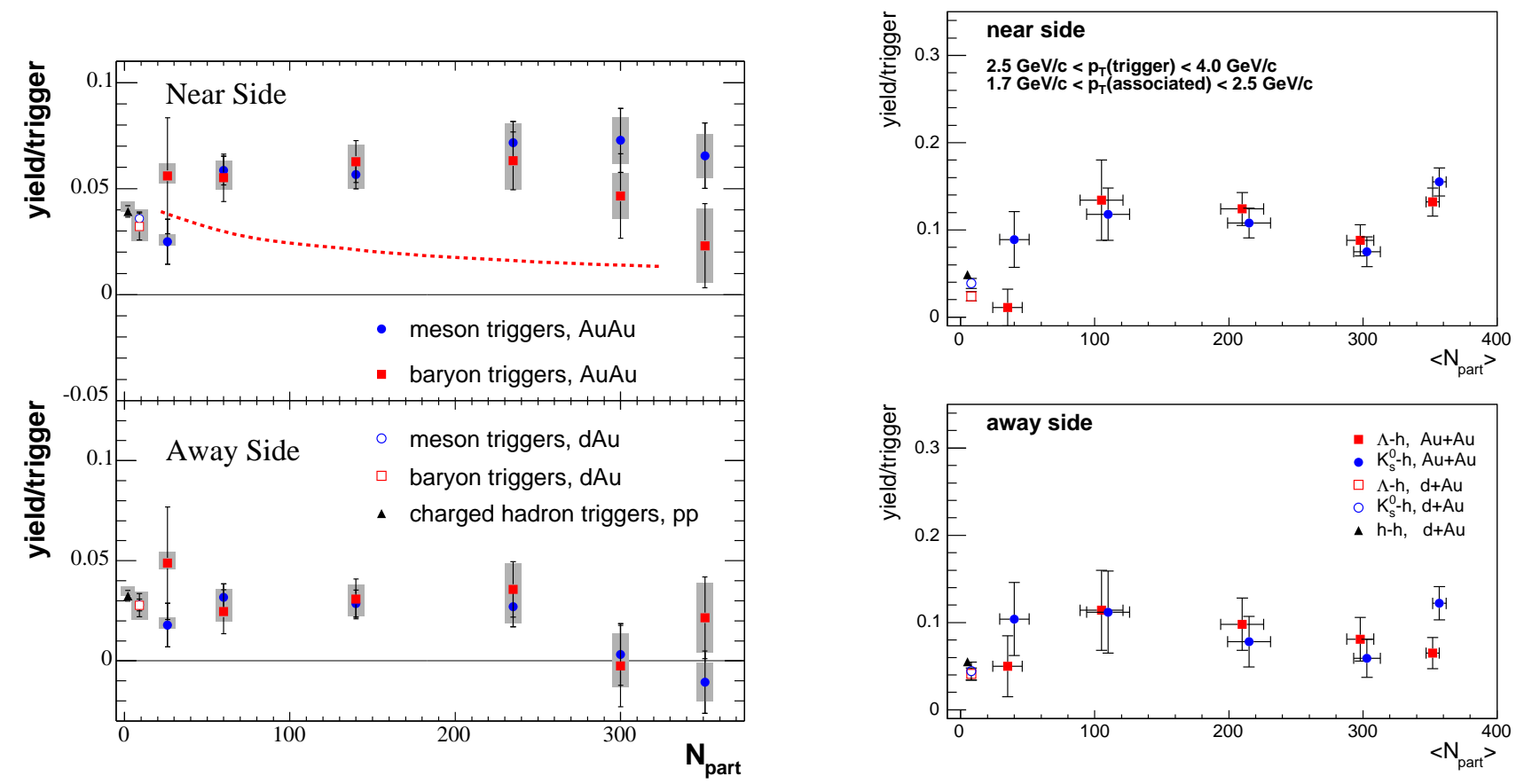

Fig. 4. Strength of dihadron azimuthal correlations with identified baryon or meson trigger and associated charged hadron, for $200 \mathrm{GeV} \mathrm{p}+\mathrm{p}, \mathrm{d}+\mathrm{Au}$ and $\mathrm{Au}+\mathrm{Au}$ collisions. Upper panels show associated yield per trigger for $\Delta \phi \sim 0$, lower panels for $\Delta \phi \sim \pi$. Left: trigger proton or pion from PHENIX 42. Dashed line is estimated upper limit of near-side partner yield from thermal recombination. Right: trigger $\Lambda$ or $K_{s}^{0}$ from STAR 4344 . The acceptance is $|\eta|<0.35$ for the left panels and $|\eta|<0.7$ for the right panels, with $2.5<p_{T}{ }^{\text {trig }}<4.0 \mathrm{GeV} / \mathrm{c}$ and $1.7<p_{T}{ }^{a s s o c}<2.5 \mathrm{GeV} / \mathrm{c}$ for all panels.

the degree of inclusive suppression, as would be expected if these hadrons are generated by jets fragmenting in vacuum. It is apparent that hadron production at intermediate $p_{T}$ is dominated neither by energy loss and fragmentation of hard-scattered partons nor by recombination from an uncorrelated thermal source. Rather, it exhibits an interplay between parton fragmentation and hadronization from the bulk medium, with their relative contributions varying as a function of $p_{T}$ 40,45]. Dynamical effects may result for instance from the excitation of the thermal medium by the partonic energy loss ("wake-field" effect) 46 or the coupling of medium-induced gluon radiation to bulk longitudinal flow [47.

Figure 5 shows new dihadron correlation data presented by STAR at this conference [44. The figure shows two dimensional $(\Delta \eta \times \Delta \phi)$ correlation functions for high $p_{T}$ charged dihadrons from $\mathrm{p}+\mathrm{p}$ (upper) and central $\mathrm{Au}+\mathrm{Au}$ (lower) collisions, corrected for finite $\eta$ acceptance. (This is in contrast to Fig. [3] where the reduced pair acceptance at large longitudinal separation $\Delta \eta$ has not been taken into account, with large $\Delta \eta$ pairs relatively suppressed as a result.) Both panels show a jet-like correlation at small angular separation $(\Delta \eta \sim 0, \Delta \phi \sim 0)$ as well as correlations back-to-back in azimuth $(\Delta \phi \sim \pi)$ but broad in $\Delta \eta$ which are presumed to result from recoil jets and, in the nuclear case, from elliptic flow. The new aspect is the additional correlation strength for central $\mathrm{Au}+\mathrm{Au}$ but not $\mathrm{p}+\mathrm{p}$ which is short range in azimuth $(\Delta \phi \sim 0)$ but long range in pseudorapidity $(\Delta \eta \sim$ large $)$, and which is stronger than the recoil correlation at $\Delta \phi \sim \pi$. Preliminary analysis suggests that this long-range component is effectively uniform within the STAR acceptance $|\eta|<1$ and is distinct from the jet-like peak, perhaps indicating an independent underlying mechanism. Near-side long-range correlations for soft hadrons with $p_{T}<2 \mathrm{GeV} / \mathrm{c}$ were first noted in [48]. The new analysis extends the observations to the region where parton fragmentation may play a signficant role. The width of the short range jet-like correlation is similar in central $\mathrm{Au}+\mathrm{Au}$ and $\mathrm{p}+\mathrm{p}$ collisions for $p_{T}{ }^{\text {trig }}>6 \mathrm{GeV} / \mathrm{c}$ but exhibits medium-induced broadening a lower $p_{T}{ }^{\text {trig }}$, while the correlated yield of the jet-like peak is independent of centrality 44, consistent with Fig. 3]

The physics underlying these near-side effects is not yet clear. The long-range correlation may perhaps be due to the combined effect of the geometric trigger bias and strong transverse radial flow of the bulk [4], or coupling of induced radiation to longitudinal flow 47. Systematic studies of the $p_{T}$-dependence of the correlation will help resolve these issues. Evidence for coupling of induced radiation to bulk longitudinal flow would indicate nonnegligible energy loss, calling into question the simple picture of geometric (surface) bias of the leading particle trigger used to interpret other high $p_{T}$ data.

\section{The Future: RHIC II and the LHC}

The above results are mainly from the $2002 \mathrm{Au}+\mathrm{Au}$ run at RHIC, which delivered integrated luminosity of about $250 \mu \mathrm{b}^{-1}$. The much higher statistics 2004 dataset for 


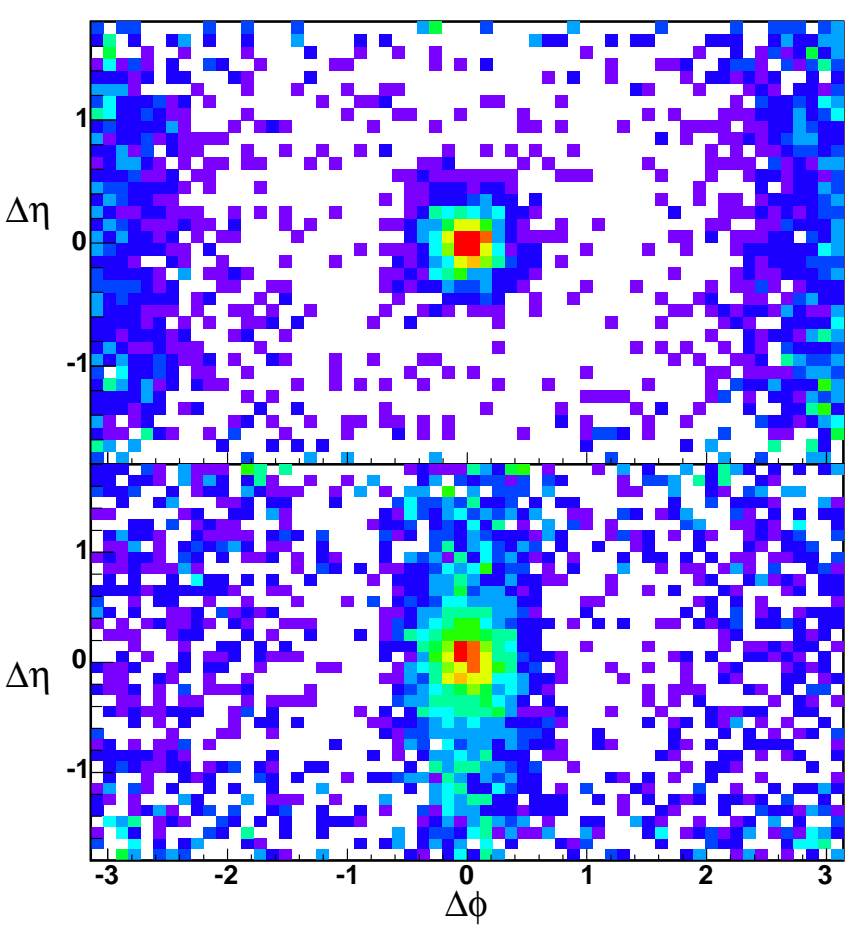

Fig. 5. Two-dimensional $(\Delta \eta \times \Delta \phi)$ charged dihadron correlation functions from minimum bias $\mathrm{p}+\mathrm{p}$ (upper) and central $\mathrm{Au}+\mathrm{Au}$ (lower) collisions at $\sqrt{s}=200 \mathrm{GeV}$, with $3<p_{T}{ }^{\text {trig }}<6$ $\mathrm{GeV} / \mathrm{c}$ and $2<p_{T}^{\text {assoc }}<p_{T}{ }^{\text {trig }} 44$.

$\mathrm{Au}+\mathrm{Au}$ at $200 \mathrm{GeV}$ now being analysed has $\sim 3.7 \mathrm{nb}^{-1}$, corresponding to about twice the RHIC design luminosity [50]. Significant RHIC detector upgrades over the next five years are aimed at improved vertexing for charm measurements, better particle identification, and low-mass dilepton measurements [50; and expanded forward coverage to address low $x$ physics [5]. The five year run plan calls for collisions of lighter ions, extensive runs with lower energy $\mathrm{Au}+\mathrm{Au}$, polarized $\mathrm{p}+\mathrm{p}$ and $\mathrm{d}+\mathrm{Au}$, and another long $200 \mathrm{GeV} \mathrm{Au}+\mathrm{Au}$ run once major detector upgrades are in place. In the longer term, a significant accelerator upgrade based on electron cooling is planned (RHIC II) which will give annual integrated luminosity for $200 \mathrm{GeV} \mathrm{Au+Au} \mathrm{of}$ $\sim 80-90 \mathrm{nb}^{-1}$. Additional upgrades to the existing large detectors and an entirely new detector are being considered to exploit the increased luminosity [50] which will extend jet-related measurements to much higher $p_{T}$, far into the perturbative regime. The broad scientific case for RHIC II is under discussion 51, but for hard probes the increased capabilities will enable much more robust $\gamma+$ jet measurements and detailed study of the charm and, in particular, beauty sectors.

First $\mathrm{p}+\mathrm{p}$ collisions at the LHC are currently expected in 2007, with first $\mathrm{Pb}+\mathrm{Pb}$ collisons at $5.5 \mathrm{TeV}$ in 2008. Jet rates at the LHC are large 52 . The nominal annual integrated luminosity for $5.5 \mathrm{TeV} \mathrm{Pb}+\mathrm{Pb}$ collisions is 0.5 $\mathrm{nb}^{-1}$, yielding $\sim 1.4 \times 10^{6}$ jets with $E_{T}>150 \mathrm{GeV}$ and $\sim 3.7 \times 10^{5}$ jets with $E_{T}>200 \mathrm{GeV}$ within acceptance $|\eta|<2.5$. The broad kinematic and statistical reach of jet production at the LHC enables study over a very broad range of fragmentation $z$ of the softening and transverse heating of jets due to interactions in the medium [53.54. Partonic energy loss is expected to vary at most logarithmically with jet energy, and the wide range of jet $E_{T}$ will provide sufficient lever arm for a stringent test of energy loss theory. Nearly complete reconstruction of jets may be possible in LHC heavy ion collisions. Complete jet reconstruction would be free of the geometric and fragmentation biases intrinsic to leading particle triggers and would be sensitive to the full $A^{2}$-scaled jet cross section, giving access to the full spectrum of energy loss in the medium.

While the identification of hard jets at RHIC II and the LHC will be straightforward, full jet reconstruction with good energy resolution must contend with the complexity of the underlying background in heavy ion collisions. The average high $E_{T}$ jet at the Tevatron has about $80 \%$ of its energy and multiplicity within a cone of radius $R=0.3$ [55], while a $\mathrm{Pb}+\mathrm{Pb}$ event with $d N_{c h} / d \eta \sim 4000$ at the LHC will deposit $\sim 100 \mathrm{GeV}$ of background energy in the same cone. Current studies indicate that $R \sim 0.3-0.4$ gives optimal signal/background for jet reconstruction in central $\mathrm{Pb}+\mathrm{Pb}$ collisions, with correspondingly limited energy resolution [56. There are large uncertainties in the modelling of fragmentation and background fluctuations used for these estimates, however, and the first LHC data will tell us much more about jet triggering and reconstruction in nuclear collisions at 5.5 TeV.

The $\gamma+$ jet final state is expected to provide the most controlled measurement of jet quenching, since the photon does not interact with the colored medium and its $p_{T}$ balances that of the recoiling jet [57. This channel is likewise free of the geometric bias of leading particle triggers. PHENIX has measured inclusive direct photons in the heavy ion environment at RHIC, helped by the hadron suppression which reduces background [6,58. The $\gamma+$ jet coincidence measurement is statistically more demanding, however, and a robust measurement extending well above $p_{T}^{\gamma} \sim 10 \mathrm{GeV} / \mathrm{c}$ will require the RHIC II luminosity upgrade 50 . The higher $p_{T}$ is important to provide dynamic range in fragmentation $z$ and to reduce the fragmentation photon contribution [58]. Hard cross sections at the LHC are substantially larger and statistically significant $\gamma+$ jet measurements in $\mathrm{Pb}+\mathrm{Pb}$ may extend to $p_{T}^{\gamma} \sim 40 \mathrm{GeV}$. Fragmentation photons are expected to dominate the cross section in this region, however, and their contribution will have to be disentangled $[59$. $\mathrm{Z}+$ jet provides a very clean but statistically more limited alternative 60 .

A crucial test of the partonic energy loss picture is the variation of energy loss with parton species. Generically, gluons should lose more energy than quarks due to their larger color charge. It has also been proposed that heavy quarks should experience smaller energy loss than light quarks due to the mass-dependent suppression of forward radiation ("dead cone effect" 61,62,63,64). Isolation of specifically quark or gluon jet effects at RHIC has proven difficult thus far, but forward triggering at moderately high $p_{T}$ may provide a controlled bias towards gluon jets [5]. Measurements of heavy quark production at RHIC 
Peter Jacobs: Jets in Nuclear Collisions: Status and Perspective

are developing rapidly 65 . It was recently shown that the suppression of D and B mesons relative to light mesons is influenced not only by the mass-dependent dead cone effect but also by color-charge dependence, especially at the LHC where gluon jets dominate light meson production 66. Measurements of D and B production over a broad $p_{T}$ range at $\mathrm{RHIC}$ and the $\mathrm{LHC}$ will map out these effects in detail.

\section{Summary}

Medium-induced modification of jet structure provides a powerful tool for the study of QCD matter created in high energy nuclear collisions. Jet modification effects at RHIC are large and statistically robust, enabling their detailed characterization. Perturbative calculations incorporating partonic energy loss via gluon bremsstrahlung agree well with the measurements, provided the medium has gluon density much greater than that of cold nuclear matter. The intermediate region $\sim 2<p_{T}<5 \mathrm{GeV} / \mathrm{c}$ exhibits an apparent interplay between fragmentation of hard partons and flow of the bulk medium that may provide a window to the partonic processes that drive the system to equilibrium. However, important aspects of energy loss theory have not yet been tested. Measurements in the near future at RHIC and the LHC will push the kinematic boundaries of jet studies significantly outward and provide strong constraints on the physics underlying partonic energy loss. It remains to be seen whether the intermediate $p_{T}$ region at the LHC will exhibit similar interplay as at RHIC between perturbative and non-perturbative processes, but it is reasonable to expect that this region will remain interesting even for fragments of the highest energy jets at RHIC II and the LHC.

\section{Acknowledgements}

I thank Urs Wiedemann for many helpful discussions.

\section{References}

1. PHENIX, K. Adcox et al., nucl-ex/0410003.

2. B. B. Back et al., nucl-ex/0410022.

3. BRAHMS, I. Arsene et al., nucl-ex/0410020.

4. STAR, J. Adams, nucl-ex/0501009.

5. L. Bland et al., hep-ex/0502040.

6. PHENIX, J. Frantz, J. Phys. G30, S1003 (2004), nuclex/0404006.

7. H. Buesching, these proceedings.

8. PHENIX, S. S. Adler et al., Phys. Rev. Lett. 91, 241803 (2003), hep-ex/0304038.

9. R. Baier, D. Schiff, and B. G. Zakharov, Ann. Rev. Nucl. Part. Sci. 50, 37 (2000), hep-ph/0002198.

10. M. Gyulassy, I. Vitev, X.-N. Wang, and B.-W. Zhang, (2003), nucl-th/0302077.

11. A. Kovner and U. A. Wiedemann, hep-ph/0304151.

12. R. Baier, Nucl. Phys. A715, 209 (2003), hep-ph/0209038.
13. K. J. Eskola, H. Honkanen, C. A. Salgado, and U. A. Wiedemann, Nucl. Phys. A747, 511 (2005), hep$\mathrm{ph} / 0406319$.

14. C. A. Salgado and U. A. Wiedemann, Phys. Rev. D68, 014008 (2003), hep-ph/0302184.

15. X.-N. Wang, these proceedings.

16. U. A. Wiedemann, hep-ph/0503119.

17. STAR, C. Adler et al., Phys. Rev. Lett. 90, 082302 (2003), nucl-ex/0210033.

18. A. Drees, H. Feng, and J. Jia, (2003), nucl-th/0310044.

19. A. Dainese, C. Loizides, and G. Paic, Eur. Phys. J. C38, 461 (2005), hep-ph/0406201.

20. D. d'Enterria, nucl-ex/0406012.

21. B. Cole, these proceedings.

22. I. Vitev and M. Gyulassy, Phys. Rev. Lett. 89, 252301 (2002), hep-ph/0209161.

23. E. Wang and X.-N. Wang, Phys. Rev. Lett. 87, 142301 (2001), nucl-th/0106043.

24. D. d'Enterria, these proceedings.

25. C. Gagliardi, these proceedings.

26. PHOBOS, B. B. Back et al., nucl-ex/0405003.

27. I. Vitev, nucl-th/0404052.

28. A. Adil and M. Gyulassy, Phys. Lett. B602, 52 (2004), nucl-th/0405036.

29. X.-N. Wang, Phys. Rev. C70, 031901 (2004), nuclth/0405029.

30. STAR, J. Adams et al., Phys. Rev. Lett. 91, 072304 (2003), nucl-ex/0306024.

31. STAR, J. Adams et al., nucl-ex/0501016.

32. N. Borghini, P. M. Dinh, and J.-Y. Ollitrault, Phys. Rev. C62, 034902 (2000), nucl-th/0004026.

33. J. Casalderrey-Solana, E. V. Shuryak, and D. Teaney, hep$\mathrm{ph} / 0411315$.

34. STAR, J. Adams et al., Phys. Rev. Lett. 93, 252301 (2004), nucl-ex/0407007.

35. PHENIX, S. S. Adler et al., Phys. Rev. Lett. 91, 172301 (2003), nucl-ex/0305036.

36. STAR, J. Adams et al., Phys. Rev. Lett. 92, 052302 (2004), nucl-ex/0306007.

37. J. Velkovska, these proceedings.

38. S. A. Voloshin, Nucl. Phys. A715, 379 (2003), nuclex/0210014.

39. R. J. Fries, B. Muller, C. Nonaka, and S. A. Bass, Phys. Rev. Lett. 90, 202303 (2003), nucl-th/0301087.

40. R. C. Hwa and C. B. Yang, Phys. Rev. C70, 024905 (2004), nucl-th/0401001.

41. V. Greco, C. M. Ko, and P. Levai, Phys. Rev. Lett. 90, 202302 (2003), nucl-th/0301093.

42. PHENIX, S. S. Adler et al., nucl-ex/0408007.

43. Y. Guo, nucl-ex/0502015.

44. D. Magestro, these proceedings.

45. V. Greco, C. M. Ko, and P. Levai, Phys. Rev. C68, 034904 (2003), nucl-th/0305024.

46. R. J. Fries, S. A. Bass, and B. Muller, nucl-th/0407102.

47. N. Armesto, C. A. Salgado, and U. A. Wiedemann, Phys. Rev. Lett. 93, 242301 (2004), hep-ph/0405301.

48. STAR, J. Adams et al., nucl-ex/0411003.

49. S. A. Voloshin, nucl-th/0312065.

50. J. W. Harris, nucl-ex/0503014.

51. http://www.bnl.gov/physics/rhicIIscience/default.asp.

52. A. Accardi et al., hep-ph/0310274.

53. C. A. Salgado and U. A. Wiedemann, Phys. Rev. Lett. 93, 042301 (2004), hep-ph/0310079. 
54. I. Vitev, hep-ph/0501255.

55. CDF, T. Affolder et al., Phys. Rev. D65, 092002 (2002).

56. A. Morsch, these proceedings.

57. X.-N. Wang and Z. Huang, Phys. Rev. C55, 3047 (1997), hep-ph/9701227.

58. PHENIX, K. Reygers, nucl-ex/0502018.

59. F. Arleo, P. Aurenche, Z. Belghobsi, and J.-P. Guillet, JHEP 11, 009 (2004), hep-ph/0410088.

60. B. Wyslouch, these proceedings.

61. Y. L. Dokshitzer and D. E. Kharzeev, Phys. Lett. B519, 199 (2001), hep-ph/0106202.

62. N. Armesto, C. A. Salgado, and U. A. Wiedemann, Phys. Rev. D69, 114003 (2004), hep-ph/0312106.

63. B.-W. Zhang, E. Wang, and X.-N. Wang, Phys. Rev. Lett. 93, 072301 (2004), nucl-th/0309040.

64. M. Djordjevic and M. Gyulassy, Nucl. Phys. A733, 265 (2004), nucl-th/0310076.

65. E. Scomparin, these proceedings.

66. N. Armesto, A. Dainese, C. A. Salgado, and U. A. Wiedemann, (2005), hep-ph/0501225. 